\title{
Simulation of the Response of the Solid State Neutron Detector for the European Spallation Source
}

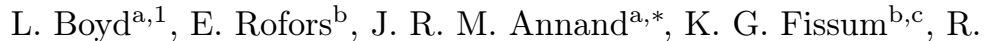 \\ Hall-Wilton $^{\mathrm{c}, \mathrm{a}, \mathrm{d}}$, R. Al Jebali ${ }^{\mathrm{a}, \mathrm{c}}$, K. Kanaki ${ }^{\mathrm{c}}$, K. Livingston ${ }^{\mathrm{a}}$, V. Maulerova ${ }^{\mathrm{b}, \mathrm{c}}$, \\ N. Mauritzson ${ }^{\mathrm{b}}$, R. Montgomery ${ }^{\mathrm{a}}, \mathrm{H}$. Perrey ${ }^{\mathrm{b}, \mathrm{c}}$, B. Seitz \\ ${ }^{a}$ School of Physics and Astronomy, University of Glasgow G12 8QQ, Scotland, UK \\ ${ }^{b}$ Division of Nuclear Physics, Lund University, SE-221 00, Lund, Sweden \\ ${ }^{c}$ Detector Group, European Spallation Source ERIC, SE-221 00 Lund, Sweden \\ ${ }^{d}$ Universita degli Studi di Milano-Bicocca, Piazza della Scienza 3, 20126, Milan, Italy
}

\begin{abstract}
The characteristics of the Solid-state Neutron Detector, under development for neutron-scattering measurements at the European Spallation Source, have been simulated with a Geant4-based computer code. The code models the interactions of thermal neutrons and ionising radiation in the ${ }^{6} \mathrm{Li}$-doped scintillating glass of the detector, the production of scintillation light and the transport of optical, scintillation photons through the the scintillator, en route to the photocathode of the attached multi-anode photomultiplier. Factors which affect the optical-photon transport, such as surface finish, pixelation of the glass sheet, provision of a front reflector and optical coupling media are compared. Predictions of the detector response are compared with measurements made with neutron and gamma-ray sources, a collimated alpha source and finely collimated beams of $2.5 \mathrm{MeV}$ protons and deuterons.
\end{abstract}

\section{Introduction}

The European Spallation Source (ESS) 1, 2] based in Lund, Sweden is currently developing a number of instruments [3] for neutron science, one of which is the Small-K Advanced DIfractometer (SKADI) [4]. This small-angle neutronscattering instrument will be used to investigate the properties of materials used in a broad range of scientific and medical research. The detector associated with the SKADI instrument is the Solid-state Neutron Detector (SoNDe) [5. 6. 7] which has a total area of $\sim 1040 \times 1040 \mathrm{~mm}^{2}$, and is constructed

\footnotetext{
* Corresponding author

Email address: john.annand@glasgow.ac.uk (J. R. M. Annand)

${ }^{1}$ Present Address: HM Naval Base Clyde Off Site Centre, Rhu, Helensburgh, G84 8NE, UK.
}

Preprint submitted to Nucl.Instr.Meth. A

12th March 2021 
from $40052 \times 52 \mathrm{~mm}^{2}$ elements. Each element consists of a $50 \times 50 \times 1 \mathrm{~mm}^{3}$ sheet of GS20 [8] ${ }^{6}$ Li-doped glass scintillator coupled optically to a Hamamatsu H12700A $8 \times 8$ pixel, multi-anode photomultiplier tube (MAPMT) [9]. With this optical sensor, it is envisioned that the neutron-interaction position resolution will be $\sim 6 \mathrm{~mm}$, the dimension of a single MAPMT pixel. The module is designed to detect thermal neutrons through neutron capture within the scintillator $n+{ }^{6} \mathrm{Li} \rightarrow{ }^{3} \mathrm{H}+{ }^{4} \mathrm{He}$. The alpha particle and triton, with a total energy of $4.78 \mathrm{MeV}$, produce optical scintillation photons which are detected by the MAPMT. A small self-contained data-acquisition (DAQ) system [10], attached directly to the rear of the MAPMT, digitises the MAPMT anode signals to produce a data set containing the amplitude, position and timing of the scintillation signal.

Various configurations of the module including:

- the use of a pixelated GS20 sheet

- the use of optical coupling medium

- the provision of a front reflector

- imperfect surface finish of the GS20 sheet

have been simulated to gauge their effect on the transport of optical photons. The response of the detector module has been measured using collimated beams of protons and deuterons [11, alpha particles [12, thermal neutrons [13] and uncollimated fast-neutron and gamma-ray sources. These measurements are compared with the predictions of the computer model described in this paper.

\section{The Computer Model}

The computer model of a SoNDe module uses the Geant4 Monte Carlo toolkit, version 4.10.6, 14 and is coded in $\mathrm{C}++$. Included in the model are the GS20 sheet, the glass of the MAPMT window, the photocathode, optional optical coupling media between the scintillator and MAPMT and optional front reflectors.

\subsection{Module Geometry}

A rendering of the geometry encoded in the computer model is displayed in Fig. 1. This shows the scintillator, with optional grooving etched into the surface of the glass. Also displayed is the borosilicate glass of the MAPMT window and the tracks of optical photons (red lines, Fig. 1(C)) started at the mid point of pixel P28. The origin of the coordinate system, shown by the cross in Fig. 1A, is the centre of the cuboid volume which defines the GS20 sheet.

The dimensions of the GS20 sheet have been set to $50.0 \times 50.0 \times 1.0 \mathrm{~mm}^{3}$ to match the size of the piece used in test measurements. A thickness of $1 \mathrm{~mm}$ produces a 
thermal-neutron detection efficiency of $\sim 76 \%$. The effectiveness of dividing the GS20 sheets into pixels to reduce the spread of signal was investigated. Grooves in the glass surface, following the pixel boundaries of the MAPMT with variable depth and width, could be introduced optionally. The default surface finish of the GS20 sheet was polished and optional polished $\mathrm{Al}$ or matt $\mathrm{TiO}_{2}$ reflector were included at the external front face of the sheet.

The dimensions of the H12700A MAPMT were taken from Ref. [9]. Only the glass window, dimensions $52.0 \times 52.0 \times 1.5 \mathrm{~mm}^{3}$, and the bialkalai photocathode, dimensions $48.5 \times 48.5 \mathrm{~mm}^{2}$, deposited on the back side of the window are modelled. Metalic side walls and internal electrode structures were not included in the model.

Although nominally flat, the borosilicate glass window of the MAPMT surface is slightly depressed at the centre with respect to the edge. Measurements were made of 35 MAPMT windows, yielding a mean depression of $0.08 \mathrm{~mm}$ with a standard deviation $\sigma=0.02 \mathrm{~mm}$. The exact shape of the window surface was not determined, but where there was no coupling medium between GS20 and MAPMT in the simulation, a small air gap of thickness $0.08 \mathrm{~mm}$ was introduced between the GS20 and the borosilicate glass window of the MAPMT (Fig. 2(A)). Where a coupling medium (e.g. optical epoxy) was introduced it also had a thickness of $0.08 \mathrm{~mm}$. Most of the calculations described in Sec. 3 have been made with a constant-thickness air gap. However the effect of a nonflat MAPMT was modelled by introducing a trapesoidal depression (Fig. 2(B)) to the window surface. Comparisons of flat and non-flat windows are given in Sec. 3.1

\subsection{Optical Properties}

Optical photons were tracked [15] through the glass components of the detector as they undergo Rayleigh scattering, absorption and medium-boundary processes. Boundary processes, which were simulated using the 'glisur model' of Geant4, include:

1. dielectric to dielectric where the photon is refracted, ie transmitted through the boundary or reflected at the boundary.

2. dielectric to metal where the photon is absorbed in the 'metal' or reflected at the boundary.

3. dielectric to black where the photon is absorbed at the boundary.

The surfaces of the GS20, borosilicate PMT glass and any optional coupling medium were assumed to be polished, so that reflection was specular, but the effect of small surface irregularities was also calculated via the 'polish' parameter incorporated in the glisur model [16, 17]. This is also investigated in Sec. 4.2.

The optical properties of each material such as refractive index, 1/e attenuation length and reflectivity have been entered, for photon energies in the range $2.066 \mathrm{eV}(600 \mathrm{~nm})$ to $4.133 \mathrm{eV}(300 \mathrm{~nm})$, into the properties tables linked to particular materials. Some of the employed optical parameters are displayed in 


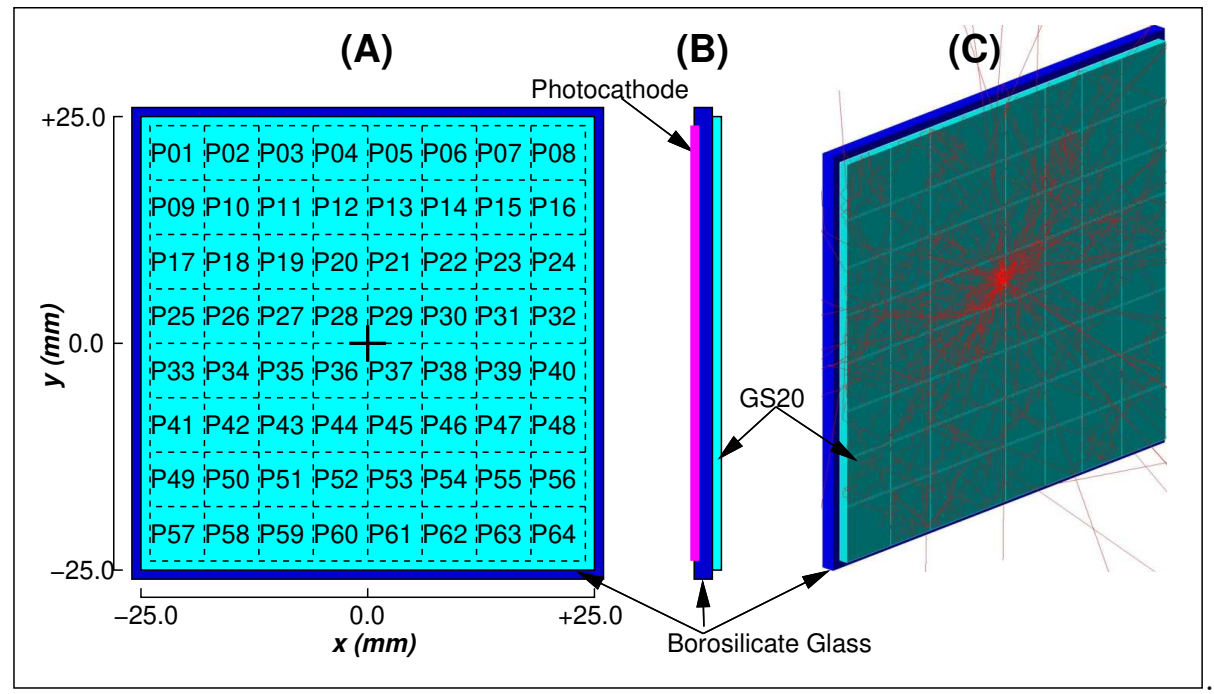

Figure 1: Rendering of the Geant4 model of a SoNDe module. (A) Front view where the cross denotes the $\mathrm{x}-\mathrm{y}$ coordinate origin and the dashed lines show the photocathode pixel boundaries. The $\mathrm{z}$ origin is at the centre of the GS20 sheet. (B) Side view showing the photocathode (magenta line) attached to the rear face of the glass window of the MAPMT. (C) 3D view where the red tracks show the scattering of 100 optical photons in the glass components.

Fig. 3. EJ500 [18], the optional optical-coupling epoxy, was given a constant refractive index of 1.57 .

A light output of $20 \%$ of anthracene (3500 photons $/ \mathrm{MeV}$ ), and a Birks parameter [19] $k_{B}=0.01 \mathrm{~mm} / \mathrm{MeV}$ has been used for GS20 scintillator. The Birks parameter models the non-linear (with respect to energy deposit) response of the scintillator, which is due to increased quenching of the scintillation signal when $d E / d x$ and the ionisation density along a charged track are large (see Eq. 21. The choice of Birks parameter value is discussed in Sec. 4.3.3

The optional outer reflector scintillator coatings were either matt $\mathrm{TiO}_{2}$ paint [20] or polished $\mathrm{Al}$ foil. The former has $\sim 91 \%$ reflectance at $390 \mathrm{~nm}$, falling to $28 \%$ at $350 \mathrm{~nm}$, while the latter has a reflectance of $92 \%$ at $390 \mathrm{~nm}$, with a small wavelength dependence [21].

\subsection{Modes of operation}

The code may be run in three different modes which are described below. In all cases information has been recorded event-by-event in ROOT TTree format 24] and then analysed using a ROOT $\mathrm{C}++$ macro.

\subsubsection{Optical Photon Transport}

Optical photons were generated isotropically at a wavelength of $395 \mathrm{~nm}(3.15$ $\mathrm{eV}$ ), at a chosen point inside the GS20 sheet, using the General Particle Source 


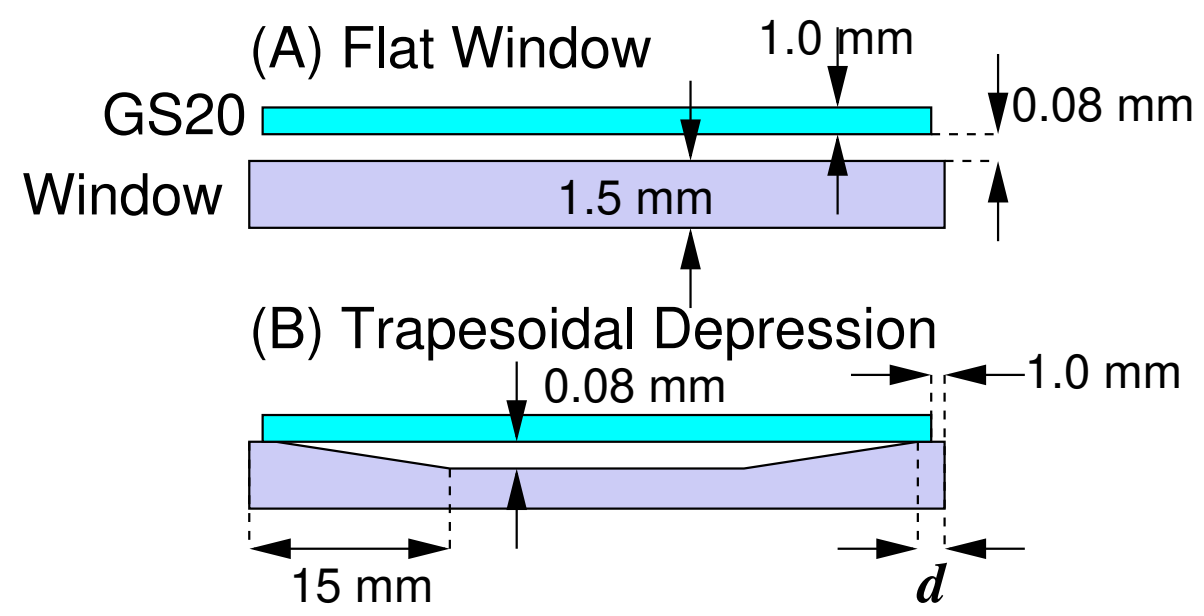

Figure 2: MAPMT window detail (not to scale).

tool of Geant4. The wavelength corresponds to the peak of the scintillation emission spectrum of GS20. When a photon impinged on the photocathode, where it was absorbed, the photon wavelength, direction and interaction position were recorded. Otherwise if absorbed outside the photocathode or scattered outside of the detector volume the photon was lost.

\subsubsection{Interactions of Ionising Radiation in GS20}

The interactions of ionisating radiation within the sheet of GS20 have been modelled using the high-precision hadronic interaction class FTFP_Bert_HP and electromagnetic interaction classes G4EmStandardPhysics and G4EmExtraPhysics. For incident neutron energies below $4.0 \mathrm{eV}$ the interaction class G4NeutronHPThermalScattering with data base G4NeutronHPThermalScatteringData was employed.

Primary and any subsequent (from gamma rays or neutrons) charged particles were tracked and the energy losses through ionisation recorded. At each discrete step along a charged-particle track the energy loss along the step, the time with respect to particle production at source and the mean step position were recorded in the ROOT TTree.

\subsubsection{Full Calculation}

This mode proceeded as in Sec. 2.3.2, but included the production of scintillation photons along the charged-particle tracks. Cherenkov radiation was also produced if the charged-particle velocity was above threshold for a particular optical medium. The optical photons were then tracked as in Sec. 2.3.1. In this mode, information for both the charged-particle track in the GS20 sheet and the optical photon impinging on the photocathode was recorded. 

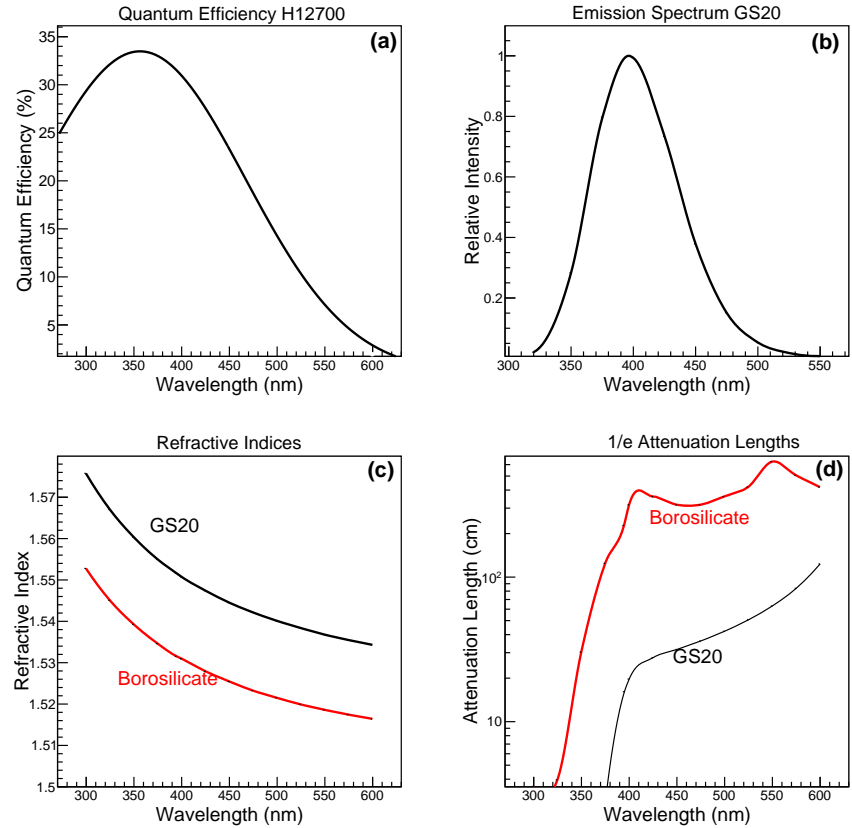

Figure 3: Optical properties employed in the simulation: (a) the quantum efficiency of the bialkalai cathode of the H12700 PMT 9, (b) the scintillation emission spectrum of GS20 22, (c) refractive indices of GS20 222 and borosilicate glass 23, (d) 1/e attenuation lengths of GS20 22] and borosilicate glass [23.

\section{Optical Photon Transport Simulations}

The transport of optical photons was simulated, for a variety of configurations of the SoNDe module, to calculate their spreading away from the point of origin before detection at the MAPMT photocathode. Parameters such as the grooving, optical coupling between scintillator and borosilicate glass, and reflective coatings for the scintillator were varied in various combinations. The optical photons were started at a random $x-y-z$ position within the GS20 and assigned a random direction of polarisation.

The $1 \mathrm{~mm}$ thick Li-glass sheet in front of the 4 pixels (P28, P29, P36, P37) bounding the centre of the MAPMT was seeded uniformly with scintillation photons. The volume seeded was a cuboid centred at coordinate $(0,0,0)$ (see cross in Fig. 1(A)) and the photon starting coordinates were constrainted to be within $-6<x<+6 \mathrm{~mm},-6<y<+6 \mathrm{~mm}$, and $-0.5<z<+0.5 \mathrm{~mm}$. The configuration of the SoNDe module was as in Run $R_{11}$ (introduced and described below). Each scintillation photon with a unique, randomly-generated $(x, y, z)$ start coordinate was then tracked until it struck a photocathode pixel, where its initial and final coordinates were recorded. Fig. 4 shows the results 

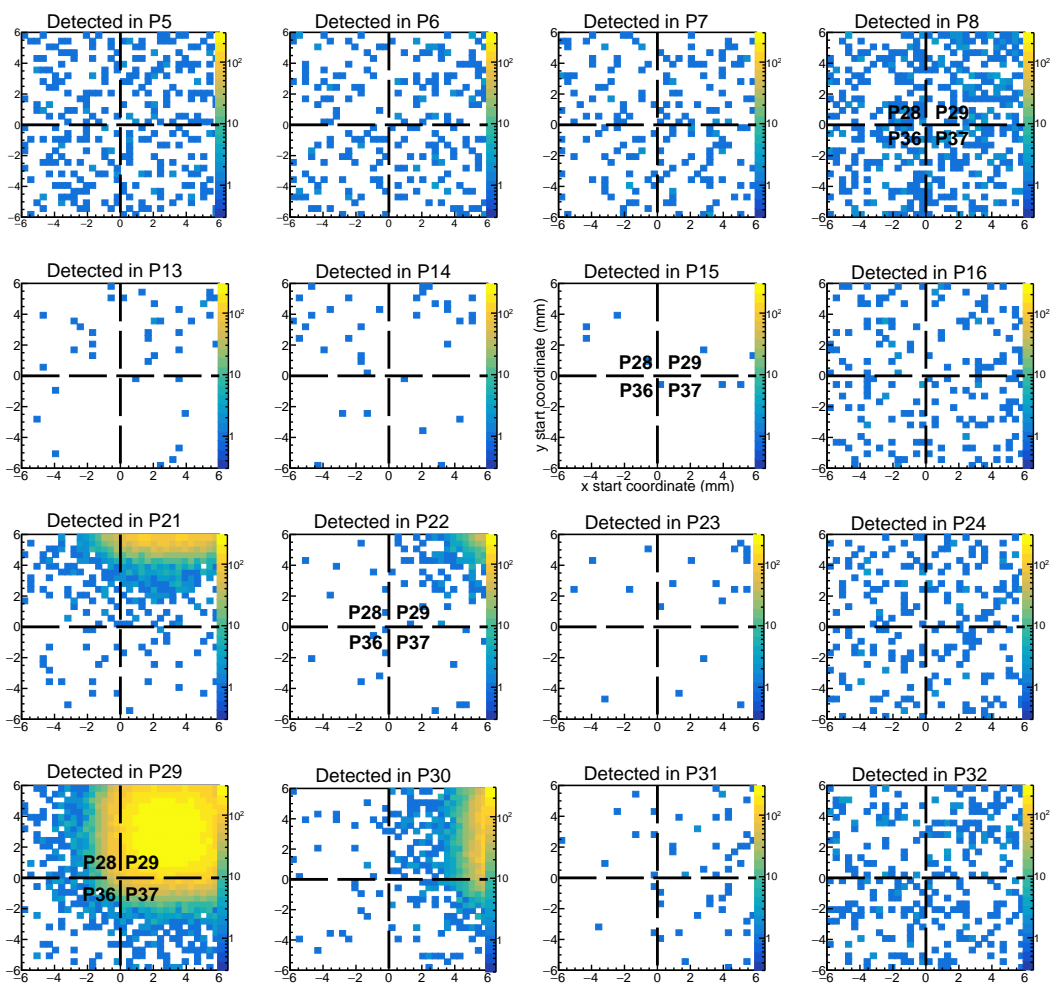

Figure 4:

Starting $(x, y)$ coordinates for photons registered in the top-right quadrant of MAPMT pixels. Dashed lines show pixel boundaries between P28, P29, P36 and P37. Pixel numbering is given in Fig. 1. Note that the $\mathrm{z}$ colour scale is logarithmic.

of this tracking, illustrating the correlation between the start coordinate of a photon and the pixel where the photon was finally detected. Each panel displays events where the photon has been detected in the corresponding photocathode pixel. The panels have been populated with the $(x, y)$ start coordinates of the photons which are detected in the corresponding pixels. The 16 pixels $\mathrm{P} 5-8$, P13 - 16, P21 - 24 and P29 - 32 correspond to the top right-hand $4 \times 4$ quadrant of the MAPMT (Fig. 1(A))

Evidently, hits in P29 were overwhelmingly due to photons seeded in front of P29, but a significant number of photons seeded in front of P28, P36 and P37, mostly within $\sim 2 \mathrm{~mm}$ of the boundary to P29, were also registered in P29. Similarly for hits in the nearest neighbours to P29: P21 (vertical), P30 (horizontal) and P22 (diagonal), the start coordinates are clustered close to the neighbour's boundary with P29. MAPMT edge pixels P5 - 8, P16, P24 and 
P32 record more photons than P13 - 15, P23 and P31, which are closer to the photon source, due to reflections at the thin side edge of the GS20 sheet. In these edge pixels, there is no obvious correlation between the start position and the pixel where the photon is detected.
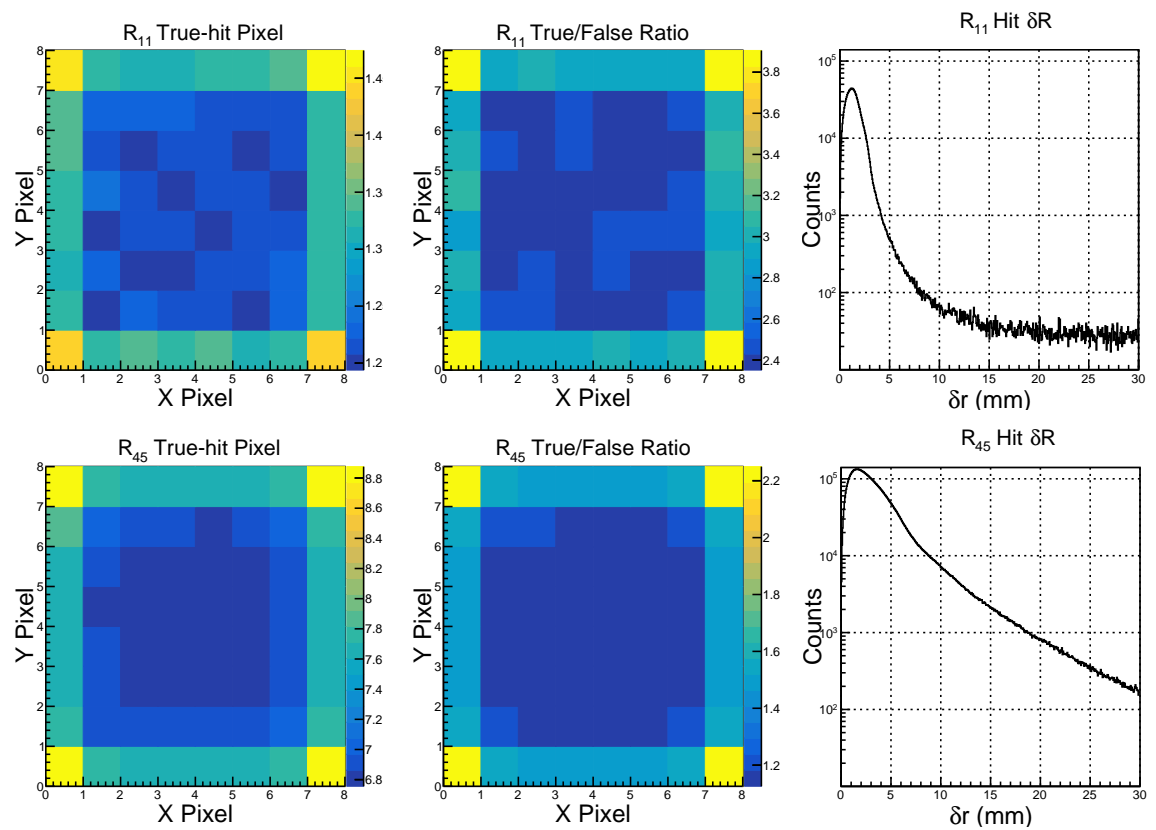

Figure 5: Top row $R_{11}$ results, left values of $\epsilon_{T}$ for each illuminated pixel, centre values of $R_{T F}$ for each illuminated pixel, right $\delta r$ for entire illuminated region. Bottom row $\mathrm{R}_{45}$ results, order as in top row. Note that the colour vertical scales are different for each $2 \mathrm{D}$ pixel plot.

Further runs were made with the $x-y$ start coordinates covering the entire GS20 sheet: $-25<x<+25 \mathrm{~mm},-25<y<+25 \mathrm{~mm}$ and again the $z$ coordinate was constrained to be within the $1 \mathrm{~mm}$ thickness. The simulation was split into 28 runs, each starting $8 \times 10^{7}$ optical photons, with direction sampled from an isotropic angular distribution. They were arranged in 4 groups of 7 . The 4 groups corresponded to conditions:

1. No grooving of the GS20 sheet.

2. Grooving $0.5 \mathrm{~mm}$ deep and $0.2 \mathrm{~mm}$ wide, cut on the outside face of the GS20 sheet.

3. Grooving $0.5 \mathrm{~mm}$ deep and $0.2 \mathrm{~mm}$ wide, cut on the inside face of the GS20 sheet.

4. Grooving $1.0 \mathrm{~mm}$ deep (i.e. all the way through the sheet) and $0.2 \mathrm{~mm}$ wide.

Within each group, the 7 sub-groups correspond to conditions:

1. No external reflector on the outside face of the GS20 sheet and no optical coupling between the GS20 and MAPMT window. 

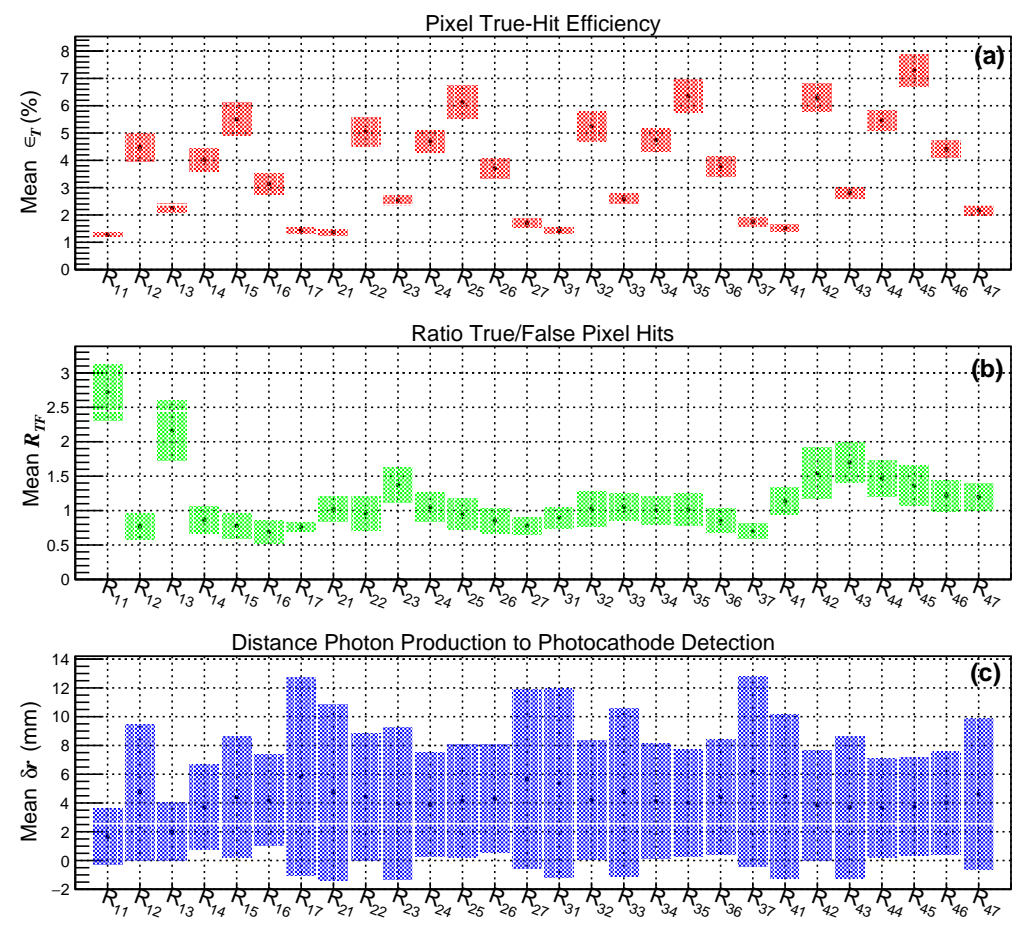

Figure 6: Summary of photon transport results: a) mean values $\epsilon_{T}$; b) mean values $R_{T F}$; c) mean values $\delta r$. The x-axis labels refer to the run labels $\mathrm{R}_{i j}$ explained in the text. The vertical extents of the filled boxes, display the standard deviations (Eq. 1).

2. $\mathrm{TiO}_{2}$ reflector on the outside face of the GS20 and no optical coupling.

3. Polished $\mathrm{Al}$ reflector on the outside face of the GS20 and no optical coupling.

4. Polished $\mathrm{Al}$ reflector on the outside face of the GS20 and optical epoxy coupling between the GS20 and MAPMT window

5. $\mathrm{TiO}_{2}$ reflector on the outside face of the GS20 and optical epoxy coupling.

6 . No reflector and optical epoxy coupling.

7. As sub-group 1, but with the surface polish factor for the GS20 sheet set to $P=0.8$. $P$ values may be varied from 0.0 to 1.0 [17] where the latter denotes a perfect surface. The MAPMT surface was set to perfectly polished $(P=1.0)$.

Runs have been labelled $\mathrm{R}_{i j}$, where $i=1,4$ is the group and $j=1,7$ is the subgroup. Analysis of the Monte Carlo output has determined the $x-y$ translation distance, $\delta r=\sqrt{\delta x^{2}+\delta y^{2}}$, where $\delta x$ and $\delta y$ are the differences between the photon-start coordinates and the photon-detection coordinates at the MAPMT photocathode. The MAPMT hardware can resolve detected photon position down to the $6 \times 6 \mathrm{~mm}$ photocathode pixel size. Thus the analysis determines if 
the photon-start pixel is the same as the photocathode-detection pixel, which is denoted a true hit. Where the start and detection pixels are not the same, this is denoted a false hit. If $n_{T}$ and $n_{F}$ denote the numbers of true and false hits respectively within a given pixel, the true-hit efficiency $\epsilon_{T}=100 \times n_{T} /\left(n_{T}+n_{F}\right)$ is just the ratio of detected-to-started photons within a given pixel (expressed in \%), while $R_{T F}=n_{T} / n_{F}$ is the ratio of the number of true-to-false hits in a given pixel.

Representative distributions are given for runs $\mathrm{R}_{11}$ and $\mathrm{R}_{45}$ in Fig. 5, which displays the pixel distributions of $\epsilon_{T}$ and $R_{T F}$ and $\delta r$ for the entire array. While the $\epsilon_{T}$ are much larger for $\mathrm{R}_{45}$, where the $\mathrm{TiO}_{2}$ reflector increases the overall light-collection efficiency significantly, the $R_{T F}$ are significantly higher for $\mathrm{R}_{11}$ (no reflector) which correlates with a significantly smaller average $\delta r$.

A summary of the results for all runs is displayed in Fig. 6. Fig. 6(a) displays $\bar{\epsilon}_{T}$ the mean value of the $\epsilon_{T}$ averaged over all illuminated pixels. The error-bars show the standard deviations of the individual-pixel, true-hit efficiencies

$$
\sigma_{\epsilon}=\sqrt{\frac{\sum_{i=1}^{N}\left(\epsilon_{i}-\bar{\epsilon}_{T}\right)^{2}}{N-1}}
$$

where $\epsilon_{i}$ are the individual-pixel, true-hit efficiencies and $N$ is the number of illuminated pixels. Fig. 6(b) displays $\bar{R}_{T F}$ the pixel-averaged mean of the $R_{T F}$ for each run, and again the error bars denote the standard deviations of the individual-pixel ratios. Fig. 6(c) displays $\overline{\delta r}$ the mean value (averaged over all illuminated pixels) of $\delta r$ for each run, where the error bars denote the standard deviation of the mean, related to the spread in event-by-event $\delta r$ values over the entire illuminated region.

Comparing the main group runs, grooving of the GS20 sheet does increase $\bar{\epsilon}_{T}$ slightly, but compared to no grooving $\bar{R}_{T F}$ is smaller and $\overline{\delta r}$ is slightly larger on average. Within the sub-groups, $\mathrm{TiO}_{2}$ matt reflector and optical epoxy coupling produce the highest $\bar{\epsilon}_{T}$. However a polished $\mathrm{Al}$ reflector gives better $\bar{R}_{T F}$ especially if no epoxy coupling is employed. Overall, the best $\bar{R}_{T F}$ is obtained in $\mathrm{R}_{11}$ : no grooves, no reflector and no epoxy coupling, although in this case $\bar{\epsilon}_{T}$ is the lowest of all runs. This results from the mismatch of refractive indices at the GS20-air-borosilicate glass boundary, which effectively selects for transmission only photons close to perpendicular incidence, reducing the spread of detected photons from their point of origin. Thus $\bar{\epsilon}_{T}$ is reduced, while $\bar{R}_{T F}$ is increased.

Comparing $R_{11}(P=1.0)$ and $R_{17}(P=0.8)$, the latter shows a large reduction in $\bar{R}_{T F}$, a large increase in $\overline{\delta r}$ and a small increase in $\bar{\epsilon}_{T}$.

\subsection{Comparison of flat and non-flat MAPMT window}

Optical simulations were performed for 6 geometries of the MAPMT window (Fig. 2): (1) flat face; (2) trapesoidal face $d=1.00 \mathrm{~mm}$; (3) trapesoidal face $d=$ 

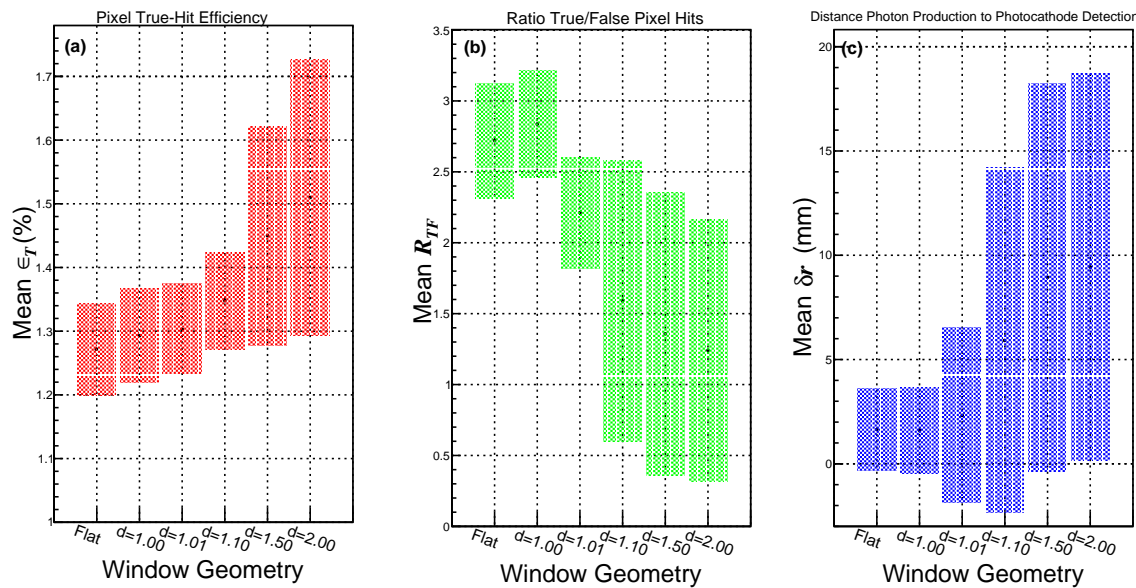

Figure 7: Window-geometry dependence: (a) true-hit efficiency $\epsilon_{T}$; (b) true-hit/false-hit ratio $R_{T F}$; (c) $\delta$ R; The vertical extents of the filled boxes, display the standard deviations (Eq. 1 ) of the respective parameters.

$1.01 \mathrm{~mm}$; (4) trapesoidal face $d=1.10 \mathrm{~mm}$; (5) trapesoidal face $d=1.50 \mathrm{~mm}$; (6) trapesoidal face $d=2.00 \mathrm{~mm}$; where the width of the overlap $(d-1.0 \mathrm{~mm})$ defines the area of GS20-glass contact at the edge of the sheet. Otherwise the SoNDe module was in $R_{11}$ configuration. The results are summerised in Fig. 7. Cases 1 and 2 are very similar, so that the shape of the air gap, trapesoidal or constant thickness, makes little difference. Where parameter $d>1.0 \mathrm{~mm}$ (cases $3-6)$, there is an area of contact between the GS20 and borosilicate window. As $d$ increases $\bar{\epsilon}_{T}$ increases slightly, $\bar{R}_{T F}$ decreases and $\overline{\delta r}$ increases. The spread of values of these parameters also increases with $d$, as the divergence in response between edge pixels, close to the contact area, and more central pixels increases.

The exact shape of the MAPMT surface has not been measured, but it seems unlikely that there will be a large contact area between the GS20 and borosilicate window. Subsequent calculations in Sec. 4 have been made with a flat MAPMT window and correspond to run case $R_{11}$ described above.

\section{Response to Ionising Radiation}

GS20 is designed to detect thermal neutrons but scintillating glass is sensitive to ionising radiation in general. The $1 \mathrm{~mm}$ thickness of the GS20 sheet is a compromise between high detection efficiency $(\sim 76 \%$, Sec. 4.3 .2$)$ for thermal neutrons and low detection efficiency for background radiation. Increasing the thickness will increase the background efficiency preferentially, relative to that of thermal neutrons. In operation at ESS the potential sources of background will be gamma rays [25] and fast neutrons [26, which will range in energy from sub-MeV to around $\mathrm{GeV}$. 
When comparing the response of a scintillator to low-energy particles of different types, non-linear effects due to quenching of the scintillation process must be taken into consideration. This is performed in Geant4 using the empirical formula of Birks [19]

$$
\frac{d L}{d x}=S \frac{\frac{d E}{d x}}{1+k_{B} \frac{d E}{d x}}
$$

which relates the scintillation light yield per unit path $d L / d x$ to the scintillation efficiency $S$, the differential charged-particle energy loss $d E / d x$ and a material-dependent constant $k_{B}$, often known as the Birks parameter. This parameter is not well established for the glass GS20. A study of a variety of inorganic crystaline scintillators [27] has produced values of $k_{B}$ in the range $10^{-3}-10^{-2} \mathrm{~g} / \mathrm{cm}^{2} / \mathrm{MeV}$. A value: $k_{B}=10^{-2} \mathrm{~mm} / \mathrm{MeV}$ has been employed for GS20 (equivalent to $2.5 \times 10^{-3} \mathrm{~g} / \mathrm{cm}^{2} / \mathrm{MeV}$ ), which is discussed in Sec. 4.3 .3 .

\subsection{Position-Dependent Alpha-Particle Response}
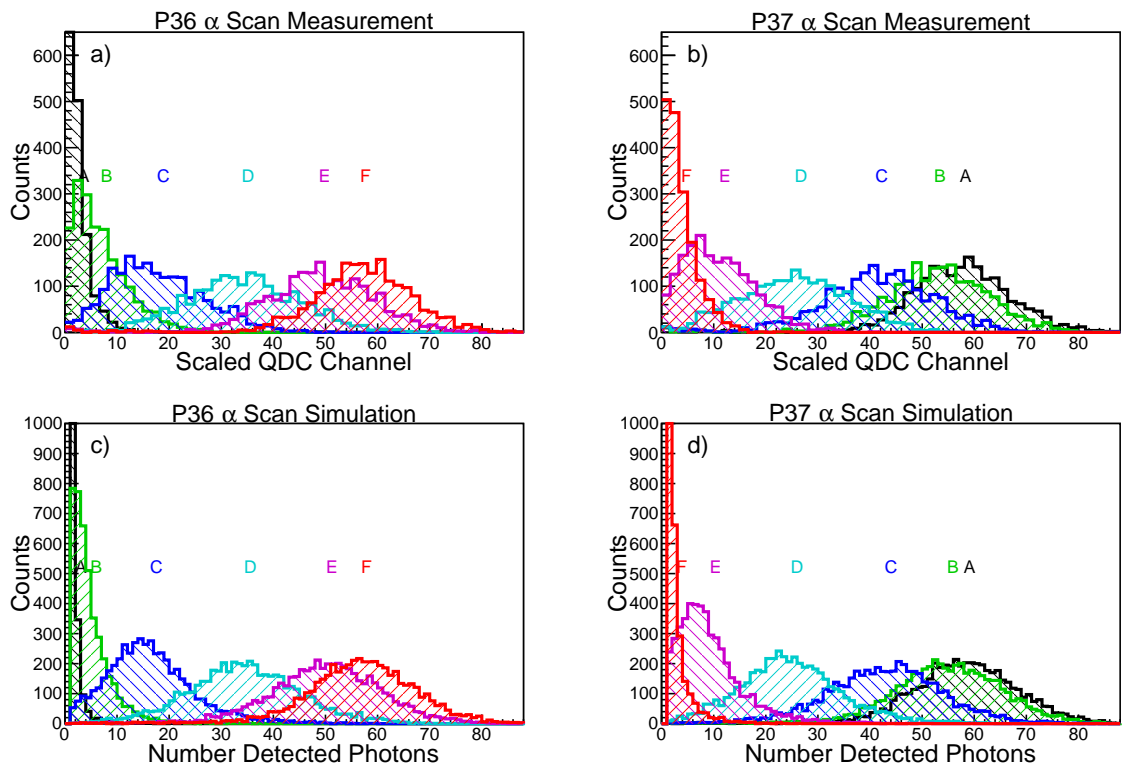

Figure 8: Alpha source horizontal scan signal amplitude spectra: a) P36 measurement; b) P37 measurement; c) P36 simulation; d) P37 simulation.

A collimated ${ }^{241} \mathrm{Am}$ alpha-particle source was employed to measure the position dependence of the signal amplitude from the individual MAPMT pixels of a SoNDe module. Measurements of the alpha response, as the collimated source was scanned across the face of the GS20 sheet, have been reported in 

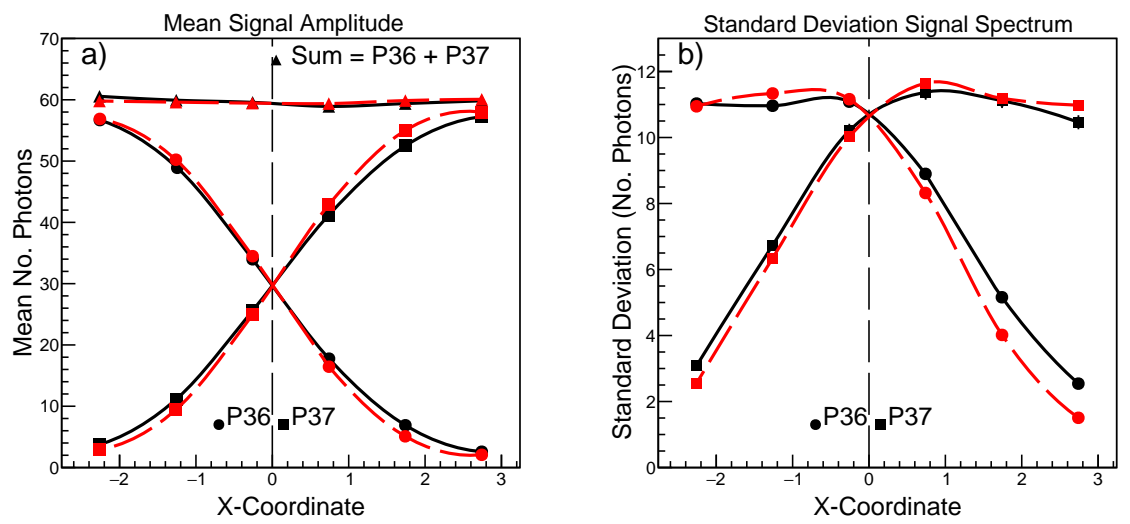

Figure 9: Alpha source scan position dependence: a) measured (black) and simulated (red) signal amplitudes; b) measured (black) and simulated (red) standard deviations of signal amplitude spectra. Circles denote pixel P36 and squares P37. The dashed line denotes the vertical boundary between P36 and P37.

Ref. [12] and these measurements have been simulated with the present computer model. Incident alpha-particle energies were sampled from the measured energy spectrum of the employed ${ }^{241} \mathrm{Am}$ source and their direction was restricted to a cone of half-angle $6.2^{\circ}$ and axis normal to the plane of the scintillator sheet, to reproduce the experimental collimation. The alpha particles passed through $\sim 6 \mathrm{~mm}$ of air before incidence on the GS20, so that their mean energy at the scintillator was $\sim 4 \mathrm{MeV}$ (see Fig. 2 of Ref. [12]) and the diameter of the illuminated spot at the GS20 face was $\sim 1.3 \mathrm{~mm}$. There was $\sim 0.2 \mathrm{~mm}$ uncertainty in the experimental beam spot diameter due to uncertainties in the internal dimensions of the metal source container.

Fig. 8 compares the measured alpha-particle spectra, from MAPMT pixels 36 and 37 (Fig. 1 (A)), to the corresponding simulated spectra for a horizontal scan over positions labeled A-F (see Fig. 5 of Ref.[12]). Labels A, B, C, D, $\mathrm{E}, \mathrm{F}$ correspond to $x$ coordinates $2.74,1.74,0.74,-0.26,-1.26,-2.26 \mathrm{~mm}$ respectively, with the $y$ coordinate fixed at $-3.4 \mathrm{~mm}$. The coordinate system is displayed in Fig. 1(A). Fig. 9 compares the position dependence of the mean values and widths of the spectral distributions at the 6 scanned $x$ coordinates. The bin widths of the measured histograms (QDC channel in Ref. [12]) have been scaled, so that the average of the 6 summed $(\mathrm{P} 36+$ P37) points is equal to the corresponding simulated quantity, i.e. the measured mean signal charge has been normalised to the calculated mean number of detected scintillation photons.

The agreement between the measured and simulated position dependence of the mean pulse height is excellent. The agreement for the position dependence of the widths (standard deviations of the spectral distributions) is also good, although the simulation slightly overpredicts the change in signal amplitude 
as one moves from position A to F. This would be expected if effects such as electronic noise, which were not simulated, made a non-negligible contribution to the measurement. This is explored further in Sec. 4.2

\subsection{Position-Dependent Response to Monoenergetic Protons}
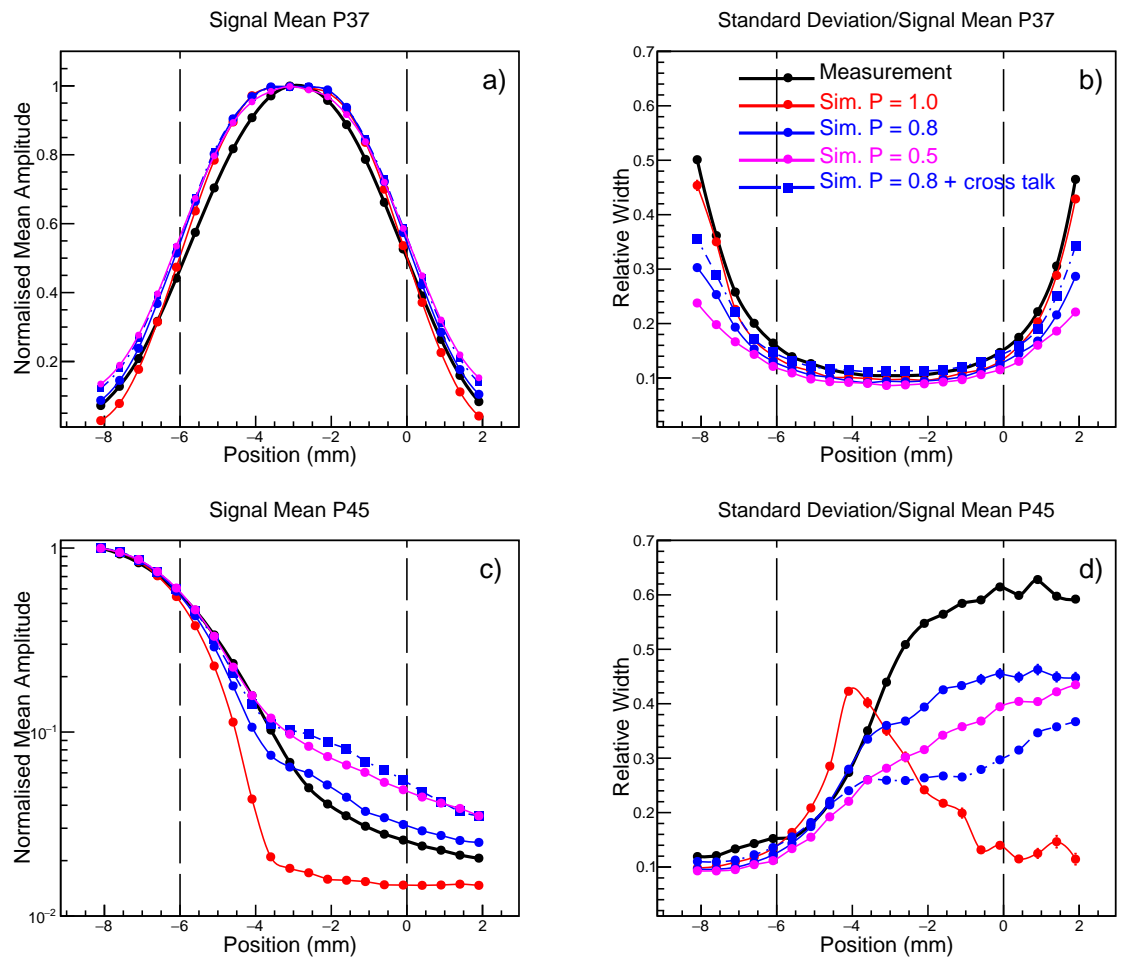

Figure 10: Vertical proton scan: means and standard deviations of pulse-height spectra from P37 (plots (a), (b)) and P45 (plots (c), (d)). Colour coding is shown in plot (b) and applies to all sub-plots. The vertical dashed lines show the boundaries of pixels 45-37 (left) and 37-29 (right).

The Lund LIBAF facility 28] provides finely-collimated, monoenergetic beams of protons and deuterons at energies up to $3 \mathrm{MeV}$. Ref. [11] describes detailed scans of LIBAF proton beams across the face of a SoNDe module and gives a first comparison of the proton measurements with the simulation. Here we show a further comparison with unpublished data taken at LIBAF and the latest version of the simulation.

Two linear scans were made: horizontal covering coordinates $2.4-12.9 \mathrm{~mm}$ at fixed $y=1.90 \mathrm{~mm}$, in $0.5 \mathrm{~mm}$ steps and vertical covering coordinates -5.6 $1.9 \mathrm{~mm}$ at fixed $x=8.4 \mathrm{~mm}$, in $0.5 \mathrm{~mm}$ steps. The beam energy was set to $2.5 \mathrm{MeV}$ and energy losses in the beam line and air before protons hit the GS20 
sheet amounted to around $20 \mathrm{keV}$. Details of the proton beam line and $\mathrm{x}-\mathrm{y}$ scanner are found in Ref. [11. The MAPMT was operated at a high voltage of $-1.0 \mathrm{kV}$ and data were collected using a VMEbus-based, data-acquisition system similar to that described in Ref. [29] with the pulse-height spectra accumulated in CAEN V792 charge-to-digital converters.

Fig. 10 compares the vertical-scan measurements (black circles and lines), for vertically adjacent pixels P37 and P45, with equivalent simulations. Horizontal scan data produced very similar distributions. The base-level simulation (red circles and lines), equivalent to case $R_{11}$ above, modeled optical transport and did not consider electronic effects. A perfectly smooth surface was assumed initially for the GS20 and MAPMT glass, which is parametrised by a 'polish' factor $P=1.0$. The effect of a non-perfect GS20 surface was then investigated with $P=0.8$ (blue circles and lines) and $P=0.5$ (magenta circles and lines). The pure optical-transport calculations were then smeared using simple parametrisations of electronic noise and cross talk in the MAPMT. The resultant distribution at $P=0.8$ is displayed with blue squares and dashed lines.

The mean amplitudes in plots a) and c), which are the mean channels of the pulse-height spectra, have been normalised so that the highest mean amplitude in each distribution is 1.0. The relative widths plotted in b) and d) are the ratios of the standard deviations and means of the pulse-height spectra.

The simple model of electronic cross talk assumes that signal leakage from one pixel to a nearest neighbour can be approximated by sampling from a Gaussian distribution centred at $3 \%$ of the signal amplitude (number of scintillation photons) with a width $(\sigma)$ of $3 \%$ of the signal amplitude. Measured cross-talk values for the H12700 30 range from $\sim 1 \%$ to $\sim 7 \%$. For each nearest neighbour, a randomly-generated leakage signal is then subtracted from the pixel signal and added to the neighbour. For pixel $P_{j}$, the nearest neighbours are $P_{j-8}, P_{j-1}, P_{j+1}, P_{j+8}$ (if the neighbour exists). Actual cross talk [30] is rather more complicated as the magnitude varies considerably from pixel to pixel, the spectrum is not Gaussian and the cause can be both misrouting of photoelectrons through the dynode chain and pickup effects at the electronic output side. None the less the model gives an idea of the magnitude of the effect.

Smearing of the signal due to noise has been sampled from a Gaussian of width $(\sigma)$ obtained from the measured widths of the pedestals of the ADC spectra [29. The widths are equivalent to $\sim 2$ detected photons and at this level the effect of noise is small compared to cross talk.

Comparing measurements with the base-level simulation, the latter underpredicts the spreading of scintillation light from its point of origin. It gives a positiondependent distribution of mean pulse height which is too flat close to a pixel centre, and too steep close to a pixel boundary. When imperfect polish is introduced, this increases the scintillation-photon spreading. A value $P=0.5$ overpredicts the spreading to adjacent pixels while $P=0.8$ is closer to the measurement, although it still gives a distribution which is too flat near the pixel centre. When $3 \%$ cross talk (see above) is introduced at $P=0.8$ the apparent signal spreading is increased significantly and is similar to the $P=0.5$ 
case with no cross talk. Thus it is difficult to separate the two effects through comparison with the present measurements.

Non-uniform sensitivity of the photocathode, as investigated for the similar H8500 MAPMT [31, is a further potential source of distortion of the measured response. It has not been included in the present calculations, pending a better knowledge of the sensitivity of the employed H12700A MAPMT.

\subsection{Simulation of Potential Background}

Fast neutrons and gamma rays are a potential source of background at the ESS. Here the measured and simulated response of a SoNDe module to these backgrounds is presented. The measurements used the same experimental setup as for the proton scan (Sec. 4.2 and were made on the same day using the facilities of the Lund University Source Test Facility [32].

\subsubsection{Neutron and Gamma-ray Source Measurements}

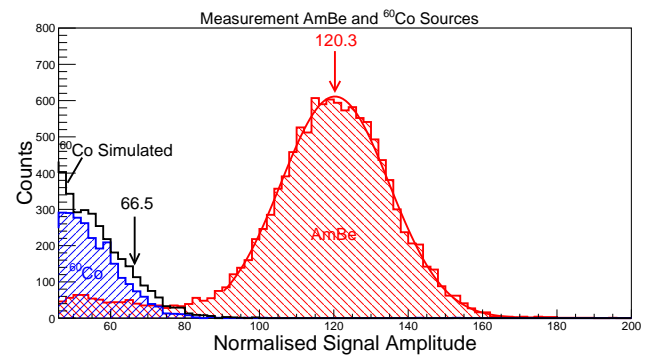

Figure 11: Pulse Height Measurement. Blue: ${ }^{60}$ Co gamma-ray source, red: AmBe neutron and gamma-ray source. The smooth curve is a Gaussian fit to the measured neutron-capture peak. The black line shows the end-point of the simulated ${ }^{60}$ Co spectrum.

The SoNDe module was irradiated with AmBe neutron and ${ }^{60}$ Co gamma-ray sources. A Pb brick of thickness $20 \mathrm{~mm}$, placed between the AmBe source and

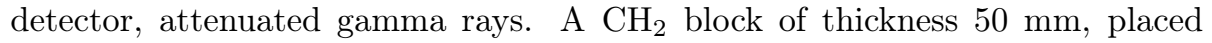
detector-side of the $\mathrm{Pb}$, provided some moderation of the fast neutrons. The signal amplitude (Fig. 11) has been reconstructed from a nine-pixel cluster sum centred on pixel P37. The AmBe run shows a prominant thermal-capture peak (energy $4.78 \mathrm{MeV}$ ) at channel 120.3, which has been normalised to coincide with the simulated number of detected scintillation photons. Measurements of the SoNDe response to a collimated thermal neutron-beam produced at a reactor [13] show that the capture peak is very similar to that obtained with AmBe. Background in the AmBe spectrum from fast neutrons and gamma rays does not pollute the neutron-capture peak significantly. This peak may thus be compared to that of the thermal-neutron simulation (Fig. 12), which does not include the fore-mentioned background. 
The ${ }^{60}$ Co spectrum shows the end point of the smeared Compton distribution and compares it to the simulated distribution (Sec. 4.3.2). The simulated edge (mean energy $\sim 1.0 \mathrm{MeV}$ ) from the unresolved 1.17 and $1.33 \mathrm{MeV}$ gamma-rays aligns with channel 66.5 while the slope of the measured spectrum, normalised on the same basis as the neutron-capture peak, falls systematically $\sim 4$ channels lower. Thus the edge position for the measured ${ }^{60} \mathrm{Co}$ spectrum is estimated to fall at channel $63 \pm 6$.

Compared to Compton electrons, the alpha-triton final state obviously produces less scintillation light per unit energy deposited, which is modeled in the simulation via the Birks formalism (Eq. 2).

\subsubsection{Simulated Neutron and Gamma-ray Response}
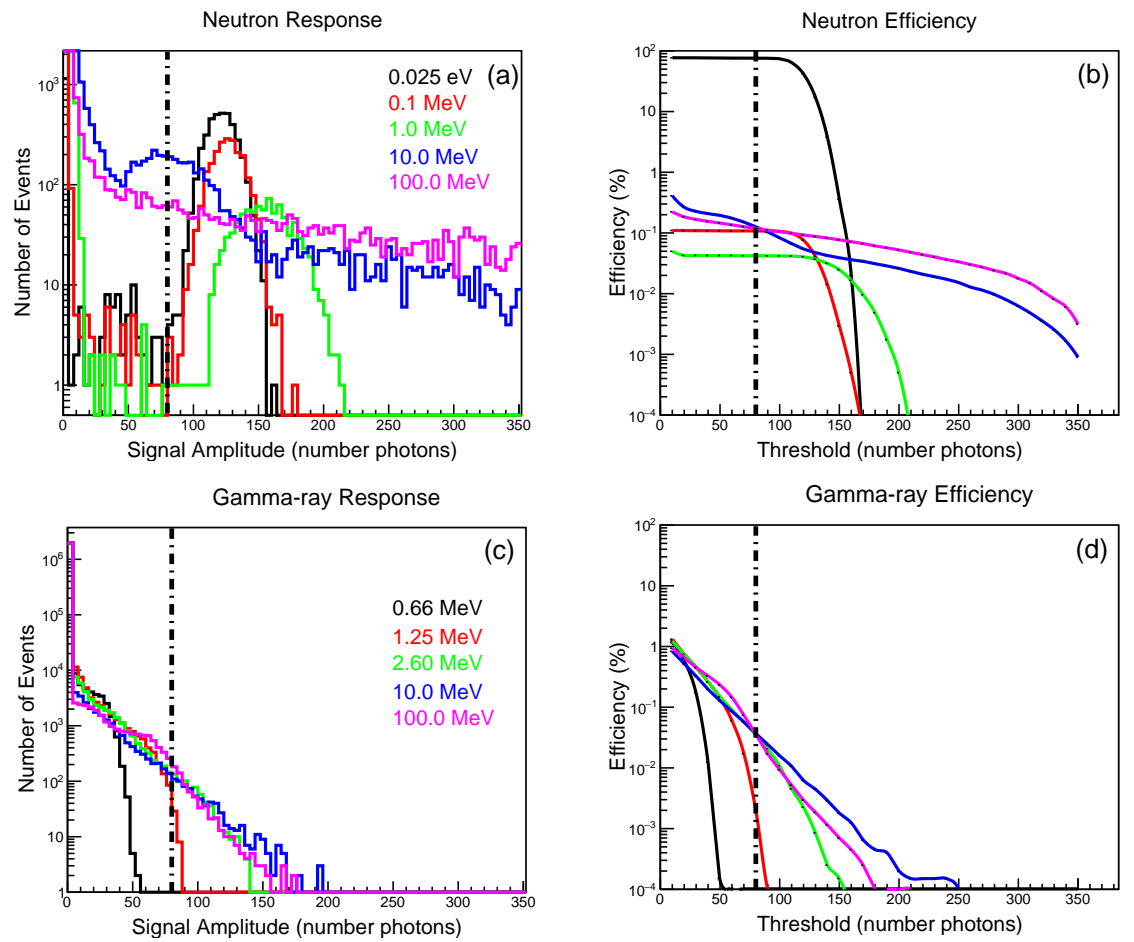

Figure 12: (a) Fast-neutron, signal amplitude compared to that of $0.025 \mathrm{eV}$ neutrons. (b) Neutron efficiency as a function of threshold. The colour coding is as for plot (a). (c) Gammaray signal amplitudes for a range of incident energies. (d) Gamma-ray efficiency as a function of threshold where the colour code is as plot (c). The dot-dash line in all panels marks a threshold level of 80 photons. Note that the thermal-neutron spectrum resulted from $5 \times 10^{3}$ incident particles, while the fast-neutron and gamma-ray spectra resulted from $2 \times 10^{6}$ incident particles.

The simulated signal amplitude, in terms of number of scintillation photons, for fast neutrons at incident energies from $0.1 \mathrm{MeV}$ to $100 \mathrm{MeV}$ is compared 
to the thermal-neutron $(0.025 \mathrm{eV})$ response in Fig. 12 (a). The displayed pulse heights are cluster sums of the 9 pixel signal amplitudes centred on P37. At low energies, where the fast neutron has insufficient energy to fragment the nuclei of the glass, there is a weak signal from neutron capture on ${ }^{6} \mathrm{Li}$. Where the neutron has sufficient energy to break up nuclei, a continuum emerges from the interactions of final state protons and light ions, becoming more pronounced as the energy increases.

Neutron detection efficiency, defined as the ratio of integrated counts above threshold to the total number of incident neutrons, is displayed as a function of threshold in Fig. 12(b). The 80-photon threshold level results in insignificant loss of thermal efficiency (76\%). At fast-neutron energies between $0.1,1.0 \mathrm{MeV}$, this threshold does not give significant background suppression, while at higher energies it offers limited suppression. Neutron detection efficiency at the 80photon threshold is given in Table 1. for a range of energies. A fast-neutron efficiency of around $0.1 \%$ is similar to that obtained with a $2.54 \mathrm{~cm}$ diameter ${ }^{3} \mathrm{He}$ proportional counter [33, 34] operating at 10 bar, but around a factor $10^{2}$ larger than the ${ }^{10} \mathrm{~B}$-coated 'Multi Blade' detector [26] developed for ESS.

The signal-amplitude spectra of the SoNDe module at incident gamma-ray energies from $0.66 \mathrm{MeV}$ to $100 \mathrm{MeV}$ have also been simulated. Fig. 12(c) displays the spectra, in terms of number of scintillation photons, where the signal amplitude was reconstructed from a nine-pixel cluster sum, as for neutrons. Gamma-ray spectra show a quite diffuse Compton edge up to energies $\sim 1 \mathrm{MeV}$, but at higher energies secondary electrons from Compton or pair-production processes have too much energy to stop in $\sim 1 \mathrm{~mm}$ of glass and the distributions appear quite similar from 2.6 up to $100 \mathrm{MeV}$. As the energy increases the secondary electrons become more focused kinematically along the incident gamma ray direction (perpendicular to the face of the GS20). This tends to reduce the thickness of GS20 traversed and hence the signal amplitude, so that the $10 \mathrm{MeV}$ gamma-ray spectrum is enhanced at amplitudes in the range $120-200$ photons, compared to the $100 \mathrm{MeV}$ gamma-ray spectrum (Fig]12(c)).

Gamma-ray detection efficiencies, as a function of detection threshold, are given in Fig. 12(d). A threshold of 80 photons (Table 1) gives complete suppression of low energy $(\leq 1 \mathrm{MeV})$ gamma rays and very effective suppression at higher energies. The efficiency of $0.002 \%$ for ${ }^{60} \mathrm{Co}$ gamma rays $(1.25 \mathrm{MeV}$ average energy) appears higher than that measured for a $2.54 \mathrm{~cm}, 10$ bar ${ }^{3} \mathrm{He}$ tube [34], but here the efficiency values are extremely sensitive to applied thresholds.

The relative signal amplitude from thermal neutrons and gamma rays has been calculated using a Birks factor $k_{B}$ (Eq. 2 of $0.01 \mathrm{~mm} / \mathrm{MeV}$. The neutron capture peak occurs at 120.3 photons. For ${ }^{60} \mathrm{Co}$, the two gamma-ray lines have Compton edges at 0.96 and $1.12 \mathrm{MeV}$, producing mean signal amplitudes of 61 and 72 photons respectively, with the mean of the two at 66.5 photons. The simulated ratio of Compton edge to n-capture peak (0.55) is consistent with the measured ratio of $0.52 \pm 0.05$ (Sec. 4.3.1). 


\begin{tabular}{|c|c||c|c|}
\hline Gamma Source & Eff. (\%) & Neutron Source & Eff. (\%) \\
\hline \hline $0.66 \mathrm{MeV}$ & 0.0 & $0.025 \mathrm{eV}$ & 75.8 \\
\hline $1.25 \mathrm{MeV}$ & 0.002 & $0.1 \mathrm{MeV}$ & 0.108 \\
\hline $2.60 \mathrm{MeV}$ & 0.035 & $1.0 \mathrm{MeV}$ & 0.042 \\
\hline $10.0 \mathrm{MeV}$ & 0.037 & $10.0 \mathrm{MeV}$ & 0.127 \\
\hline $100.0 \mathrm{MeV}$ & 0.036 & $100.0 \mathrm{MeV}$ & 0.119 \\
\hline
\end{tabular}

Table 1: Detection efficiency for gamma rays and neutrons at a threshold of 80 scintillation photons.

\subsubsection{Comparison with proton data}

The measured neutron and gamma-ray pulse-height spectra are directly comparable with the proton data, which were collected on the same system, very close in time. For the vertical scan point closest to the centre of P37, the measured 9-element sum shows a peak at channel 101.8, while the equivalent simulated value is 99.1 .

Taking the ratio of proton-peak pulse height to neutron-capture-peak pulse height, the measured value is $0.85 \pm 0.02$, while the simulation gives values of 0.82 at $k_{B}=0.01 \mathrm{~mm} / \mathrm{MeV}$ and 0.93 at $k_{B}=0.02 \mathrm{~mm} / \mathrm{MeV}$.

Note that deuteron-beam scans are also reported in Ref. [11] and there the proton and deuteron data are directly comparable. The measured ratio of deuteron-to-proton pulse height [11] was 0.80 while the simulation gives 0.79 with $k_{B}=0.01 \mathrm{~mm} / \mathrm{MeV}$ and 0.74 with $k_{B}=0.02 \mathrm{~mm} / \mathrm{MeV}$. Thus the simulation of proton, neutron-capture, deuteron and gamma-ray pulse height is consistent with a value close to $0.01 \mathrm{~mm} / \mathrm{MeV}$. Note that direct comparison with the alpha-scan data was not possible as this was performed with a different SoNDe module and DAQ system.

\section{Summary and Conclusions}

A Geant4-based simulation of the SoNDe module, designed for thermal-neutron detection at ESS, is described. Transport of scintillation photons through the glass scintillator and MAPMT window has been used to study the spreading of the optical signal away from the interaction point in the scintillator. This was performed for a variety of scintillator grooving and optical coupling options. The likely experimental configuration, with an ungrooved scintillator and no opticalcoupling between the scintillator and MAPMT, was found to give low collection efficiency for the scintillation photons, but results in tighter containment of the detected photons close to their point of origin, a goal of the SoNDe design.

Simulations of the scintillation signal for different incident particles have been compared to equivalent measurements made with alpha-particle, gamma-ray and neutron sources, as well as beams of $2.5 \mathrm{MeV}$ protons and deuterons. 
An x-y scan, in $1 \mathrm{~mm}$ steps, of a collimated alpha-particle source across the face of a SoNDe module was simulated and compared with measurements. After collimation the illuminated 'spot' on the GS20 was around $1.3 \mathrm{~mm}$ in diameter. Excellent agreement was obtained, using the base-level simulation, on the drop in signal amplitude as the interaction position moves away from the centre of a pixel of the MAPMT. However the simulation tended to underestimate the degree of light spreading slightly.

Finer-grained position scanning, in $0.5 \mathrm{~mm}$ steps, was implemented using the LIBAF proton beam at Lund, which has a beam spot of $\sim 100 \mu$ m diameter on target. The measured position dependence of the scintillation signal shows some discrepancy with the equivalent calculation of the fall of the scintillation signal, as the interaction position is moved away from a pixel centre. The base-level simulation, which assumes perfectly polished reflecting surfaces, gives too small a variation close to the pixel centre and too rapid a fall at the pixel border. It underpredicts the amount of scintillation light spreading to adjacent pixels.

The simulation was extended to include non-perfect reflecting surfaces. As the smearing from perfect specular reflection is increased, the spreading of the optical photons from point of production also increases. Similarly, the apparent amount of signal spreading increases when the effects of electronic cross talk and noise in the MAPMT and associated electronics are folded into the simulation. Much better correspondence between simulation and measurement has been achieved using a surface polish factor $P=0.8$ and assuming that on average $3 \%$ of a pixel signal crosses to its neighbour. However a more detailed knowledge of the SoNDe module is required to quantify the relative contributions of these effects. A measurement of cross talk and photocathode sensitivity, for the actual MAPMT used in the measurements could be performed with a fine-beam laser source. Although the GS20 surface appears smooth to the naked eye, it would be desirable to investigate its structure more microscopically, or alternatively to make detailed studies of the reflection of a fine optical beam from points on the surface.

The signal from AmBe neutron and ${ }^{60}$ Co gamma-ray sources was simulated and compared to measurements with the SoNDe module. The relative strength of the signals from neutron capture and Compton scattering of gamma rays are consistent with a Birks parameter of $0.01 \mathrm{~mm} / \mathrm{MeV}$. Based on this comparison, the detection efficiency, as a function of applied pulse-height threshold, has been simulated for thermal neutrons, fast neutrons and gamma rays. A threshold equivalent to 80 detected scintillation photons gives a thermal-neutron efficiency of $75.8 \%$. Gamma rays up to $1 \mathrm{MeV}$ are not detected at this threshold and at higher energies have low efficiencies $(<0.04 \%)$. The fast-neutron efficiency is somewhat higher at the $0.1 \%$ level, which is comparable to a typical ${ }^{3} \mathrm{He}$ tube but a factor $\sim 10^{2}$ larger than a ${ }^{10} \mathrm{~B}$-coated thermal-neutron detector.

A comparison between simulation and measurement of the relative pulse height from neutron-capture, $\sim 1.0 \mathrm{MeV}$ gamma rays, $2.5 \mathrm{MeV}$ protons and $2.5 \mathrm{MeV}$ deuterons is consistent with a Birks parameter of $k_{B}=0.01 \mathrm{~mm} / \mathrm{MeV}$. However the general validity of this value for a broader range of particle types and 
differential energy loss $d E / d x$ remains to be investigated.

\section{Acknowledgements}

We thank the following organisations for supporting this work: the UK Science and Technology Facilities Council (Grant No. ST/P004458/1), the UK Engineering and Physical Sciences Research Council Centre for Doctoral Training in Intelligent Sensing and Measurement (Grant No. EP/L016753/1), the European Union via the Horizon 2020 Solid-State Neutron Detector Project (Proposal ID 654124), and the BrightnESS Project (Proposal ID 676548). We also thank the LIBAF team at Lund University for the provision and operation of their excellent proton beam. The provision of detector and electronics hard-

ware by the SoNDe collaboration and the Lund Source Test Facility is gratefully acknowledged.

\section{References}

[1] R. Garoby et al., Physica Scripta, 93(1) (2017), 014001.

[2] S. Peggs et al., ESS Technical Design Report [ESS-2013-0001]. Technical report, European Spallation Source, 042013.

[3] K. H. Andersen et al., Nucl. Instr. and Meth. A 957 (2020), 163402.

[4] S. Jaksch et al., Nucl. Instr. and Meth. A 762 (2014), 22. https://arxiv. org/abs/1403.2534

[5] S. Jaksch et al., arXiv 1707.08679, 2017. https://arxiv.org/pdf/1707. 08679

[6] S. Jaksch et al., "Recent Developments of SoNDe High-Flux Detector Project", Proc. Int. Conf. on Neutron Optics (NOP2017), JPS Conf. Proc. 22 (2018), 011019.

[7] 'Scintillator detector with high count rate', EPO patent application 102014224449.8 .

[8] GS20 scintillating glass, https://scintacor.com/products/ 6-lithium-glass/.

[9] Hamamatsu H12700 Series Flat Panel Multianode PMT assembly, https: //www.hamamatsu.com/resources/pdf/etd/H12700A_TPMH1348E.pdf

[10] IDEAS Integrated Detector Electronics AS, https://ideas.no.

[11] E. Rofors et al., Nucl. Instr. and Meth A 984 (2020), 164604.

[12] E. Rofors et al., Nucl. Instr. and Meth. A 929 (2019), 90. 
[13] E. Rofors et al., arXiv:2010.0637 [physics.ins-det] (2020).

[14] J. Allison et al., Nucl. Instr. and Meth. A 835 (2016) 186-225, and references therein

[15] Geant4 Physics Reference Manual, Release 10.6, 2020, http: //geant4-userdoc.web.cern.ch/geant4-userdoc/UsersGuides/ PhysicsReferenceManual/fo/PhysicsReferenceManual.pdf

[16] Geant4 Book for Application Developers, Release 10.6, 2020http: //geant4-userdoc.web.cern.ch/geant4-userdoc/UsersGuides/ ForToolkitDeveloper/fo/BookForToolkitDevelopers.pdf

[17] P. Gumplinger, Optical Photon Processes in Geant4, Users' Workshop at CERN, Nov. 2002.

[18] Optical Cement EJ-500, https://eljentechnology.com/products/ accessories/ej-500.

[19] J. B. Birks, The Theory and Practice of Scintillation Counting, Pergamon Press, Oxford U.K., 1964.

[20] $\mathrm{TiO}_{2}$ based reflective paint, https://eljentechnology.com/products/ accessories/ej-510-ej-520

[21] G. Hass and J. E. Waylonis, J. Opt. Soc. Am. 51 (1961), 719.

[22] M.P. Hehlen et al., J. Appl. Phys. 124 (2018),124502.

[23] Optical Glass Data Sheets, Schott AG Advanced Optics, www.schott.com/ advanced_optics.

[24] R. Brun and F. Rademakers, Nucl. Instr. and Meth. A 389 (1997) 81. http://root.cern.ch/.

[25] A. Khaplanov et al., J. Inst. 8 (2013), 10025, https://arxiv.org/abs/ 1306.6247

[26] G. Mauri et al., J. Inst. 13 (2018), 03004, https://arxiv.org/abs/1712. 05614

[27] V. I. Tretyak, Astroparticle Phys. 33 (2010), 40.

[28] Lund Ion Beam Analysis Facility (LIBAF), http://www.nuclear.lu.se/ forskning/tillaempad-kaernfysik/libaf/.

[29] J. Scherzinger et al., Appl. Radiation and Isotopes 127 (2017), 98.

[30] M. Calvi et al., J. Inst. 10 (2015), 09021, https://arxiv.org/abs/1506. 04302

[31] R. A. Montgomery et al., Nucl. Instr. and Meth. A 695 (2012), 326. 
[32] F. Messi et al., "The neutron tagging facility at Lund University", IAEA Technical Report on Modern Neutron Detection (2017), https://arxiv. org/abs/1711.10286

[33] G. Mauri et al., Eur. Phys. J. Tech. and Instr. 6 (2019), Article 3, https: //arxiv.org/abs/1902.09870v1

[34] F. Piscitelli et al., Eur. Phys. J. Plus 135 (2020), 577, https://arxiv. org/abs/2002.08153v1 